\title{
CORRECTION
}

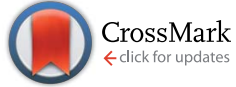

Cite this: J. Mater. Chem. C, 2015, 3 , 1142

DOI: $10.1039 / \mathrm{c} 5 \mathrm{tc} 90019 \mathrm{k}$

www.rsc.org/MaterialsC

\section{Correction: Intramolecular charge transfer (ICT) of a chiroptically active conjugated polymer showing green colour}

\author{
Hirotsugu Kawashima, Kohsuke Kawabata and Hiromasa Goto*
}

Correction for 'Intramolecular charge transfer (ICT) of a chiroptically active conjugated polymer showing green colour' by H. Kawashima et al., J. Mater. Chem. C, 2015, DOI: 10.1039/c4tc02124j.

The authors of this article were not aware at the time of its publication that the monomer they reported had previously been synthesised by Roncali et al. in 2000. ${ }^{1}$ The authors would therefore like to cite the work of Roncali et al. and apologise for this oversight. While Roncali et al. employed $\mathrm{Pd}\left(\mathrm{PPh}_{3}\right)_{2} \mathrm{Cl}_{2}$ (bis(triphenylphosphine)palladium(II) dichloride) as a $\mathrm{Pd}(\mathrm{II})$ catalyst for the Stille coupling reaction, in the J. Mater. Chem. C paper, Kawashima et al. used $\operatorname{Pd}\left(\mathrm{PPh}_{3}\right)_{4}$ (tetrakis(triphenylphosphine)palla$\operatorname{dium}(0))$ as a $\operatorname{Pd}(0)$ catalyst for the Stille coupling reaction. The authors therefore maintain that their polymerisation method in a liquid crystal medium to obtain the chiral polymer they characterised, chiral charge carriers, and the electrochemically driven change in the charge transfer band are original.

The Royal Society of Chemistry apologises for these errors and any consequent inconvenience to authors and readers.

\section{References}

1 J.-M. Raimundo, P. Blanchard, H. Brisset, S. Akoudad and J. Roncali, Chem. Commun., 2000, 939-940.

Division of Materials Science, Faculty of Pure and Applied Sciences, University of Tsukuba, Tsukuba, Ibaraki 305-8573, Japan. E-mail: gotoh@ims.tsukuba.ac.jp 